\title{
Controle químico de Salvinia molesta e monitoramento ambiental de agrotóxicos em corpos hídricos
}

\section{Chemical control of Salvinia molesta and environment monitoring of pesticides in waterbodies}

\author{
Nathalia Garlich ${ }^{1,2}$, Claudinei da Cruz $^{1}$, Robinson Antonio Pitelli ${ }^{3}$, Marcelo da Costa Ferreira ${ }^{2}$ \\ ${ }^{1}$ Centro Universitário da Fundação Educacional de Barretos (UNIFEB), Barretos. \\ ${ }^{2}$ Universidade Estadual Paulista (UNESP), Faculdade de Ciências Agrárias e Veterinárias, Jaboticabal. \\ ${ }^{3}$ Ecosafe Agricultura e Meio Ambiente, Jaboticabal.
}

\begin{abstract}
Resumo
As macrófitas são importantes nos ambientes aquáticos. Entretanto, devido às ações antrópicas, os corpos hídricos tornam-se eutrofizados, o que promove o aumento de algumas espécies em detrimento de outras. A Salvinia molesta é uma macrófita aquática flutuante e tem causado muitos prejuízos para os usos múltiplos da água e o seu crescimento excessivo tem contribuído para a diminuição da qualidade de água nos corpos hídricos em diversas regiões do mundo. Uma maneira de conter seu crescimento é a aplicação de herbicidas, que têm sido muito utilizados em vários países. Um dos principais questionamentos sobre a utilização de herbicida em ambientes aquáticos é o efeito da decomposição da matéria morta vegetal no interior do corpo hídrico, o que pode interferir na qualidade de água e o efeito da aplicação para organismos não alvos. Portanto, para a aplicação de produtos químicos nos ambientes aquáticos, são necessários estudos de simulação ambiental para avaliar o impacto da utilização e o potencial risco ambiental ocorrido durante e após o processo de intervenção química. O objetivo deste estudo foi discorrer sobre o controle químico de S. molesta no ambiente aquático e a importância do monitoramento ambiental dos corpos hídricos por meio da revisão de literatura. As literaturas analisadas mostraram que o estudo de tecnologias para aplicação de herbicidas no controle de S. molesta são importantes, para manter a planta em nível populacional que não cause prejuízos aos corpos hídricos. E que estudos de simulação ambiental podem auxiliar na padronização da aplicação e no monitoramento do ambiente para que ocorra eficiência no controle das plantas alvo e segurança aos organismos aquáticos.
\end{abstract}

Palavras-chave: planta aquática; manejo; tecnologia de aplicação; aplicação de herbicidas; sanidade; qualidade de água; ambiente aquático.

\begin{abstract}
Macrophytes are important in aquatic environments. However, due to anthropic actions, waterbodies become eutrophic, which promotes the increase of some species to the detriment of others. Salvinia molesta is a floating aquatic macrophyte and has caused many losses for the multiple uses of water and its excessive growth has contributed to the decrease in water quality in waterbodies from different regions of the world. One way to curb its growth is the application of herbicides that have been widely used in several countries. One of the main questions about the use of herbicide in aquatic environments is the effect of decomposition of dead plant matter inside the waterbody, which can interfere with water quality and the effect of application to non-target organisms. Therefore, for the application of chemicals in aquatic environments, environmental simulation studies are needed to assess the impact of use and the potential environmental risk that occurred during and after the chemical intervention process. The objective of
\end{abstract}

Autor para correspondência: Profa. Dra. Nathalia Garlich, e-mail: nathalia.garlich@gmail.com Recebido para publicação: 23/10/2020

Aceito para publicação: 13/05/2021

https://doi.org/10.4322/1980-0029.162021 
this study was to discuss the chemical control S. molesta in the aquatic environment and the importance of environmental monitoring of waterbodies through a literature review. The analyzed literature showed that the study of technologies for the application of herbicides in the control of S. molesta is important, to maintain the plant at a population level that does not cause damage to waterbodies and that environmental simulation studies can assist in standardizing the application and monitoring the environment so that there is efficiency in the control of target plants and safety to aquatic organisms.

Keywords: aquatic plant; management; application technology; herbicide application; sanity; water quality; aquatic environment.

\section{Introdução}

As macrófitas são importantes nos ambientes aquáticos, se reproduzem rapidamente e disponibilizam biomassa vegetal para animais herbívoros, servem de abrigo para reprodução e proteção de organismos aquáticos e regulam as concentrações de íons dissolvidos na água (Pitelli et al., 2018). Os principais grupos de macrófitas quanto ao seu biótipo são as emersas, com folhas flutuantes, submersas, enraizadas, submersas livres e flutuantes (Esteves, 1998).

Entretanto, devido às ações antrópicas, os corpos hídricos tornam-se eutrofizados, o que promove o aumento de algumas espécies em detrimento de outras, formando colonizações de plantas monoespecíficas ou pouco diversificadas, que impactam negativamente os corpos hídricos (Silva et al., 2012).

$\mathrm{O}$ seu rápido crescimento e produção de biomassa causam impactos ecológicos e econômicos nos ecossistemas aquáticos (Sartain \& Mudge, 2019), com a obstrução do fluxo de água nas turbinas em hidrelétricas, dificultam o transporte e navegação, prejudicam as atividades de esportes aquáticos, reduzem a geração de energia elétrica e a disponibilidade da água nos sistemas de irrigação e drenagem (Hussner et al., 2017), reduzem a quantidade de espécies nativas, servem de habitat para reprodução de insetos causadores de doenças e reduzem a disponibilidade de oxigênio dissolvido na água, o que pode causar a mortalidade dos organismos aquáticos (Maria et al., 2018; Sartain \& Mudge, 2019).

A Salvinia molesta é uma macrófita aquática flutuante e tem causado muitos prejuízos para os usos múltiplos da água, e o seu crescimento excessivo tem contribuído para a diminuição da qualidade de água nos corpos hídricos em diversas regiões do mundo. Uma maneira de conter seu crescimento é a aplicação de herbicidas, que tem sido muito utilizado em vários países, como a principal técnica de controle (Hussner et al., 2017; Sartain \& Mudge, 2018). Diante disso, o estudo de tecnologias de aplicação, formas de manejo, eficácia e monitoramento ambiental são importantes para o controle destas plantas aquáticas.

Um dos principais questionamentos sobre a utilização de herbicida em ambientes aquáticos é o efeito da decomposição da matéria morta vegetal no interior do corpo hídrico, o que pode interferir na qualidade de água e o efeito da aplicação para organismos não alvos pelo risco de deriva da pulverização e movimento do herbicida para fora da área de aplicação. Além disso, pelo contato direto desses organismos, uma vez que vivem no mesmo ambiente da planta a ser controlada, sendo esta a principal desvantagem da utilização de herbicidas nos ambientes aquáticos (Cruz et al., 2015a, 2015b; Garlich et al., 2016a, 2016b; Souza et al., 2017).

A aplicação de herbicida e suas tecnologias, como adjuvantes e eletrificação das gotas de pulverização em associação ou de forma sequencial, podem facilitar o controle de S. molesta e minimizar os impactos ambientais decorrentes da decomposição da macrófita e diminuir os riscos aos organismos e locais não alvos da aplicação (Santos et al., 2020; Garlich, 2020). Portanto, para a aplicação de produtos químicos nos ambientes aquáticos, são necessários estudos de simulação ambiental em mesocosmos ou microcosmos para avaliar o impacto da utilização e o potencial risco ambiental ocorrido durante e após o processo de intervenção química (Guimarães et al., 2003; Garlich, 2020).

Assim, o objetivo deste estudo foi realizar uma revisão de literatura e discorrer sobre o controle químico da macrófita aquática Salvinia molesta, com a utilização de herbicidas no ambiente aquático e a importância do monitoramento ambiental dos corpos hídricos. 


\section{Desenvolvimento}

\section{Método}

$\mathrm{O}$ artigo consiste em um estudo de revisão de literatura nacional e internacional baseado em buscas em periódicos e artigos científicos disponíveis no Sciencedirect, Scielo, Google Acadêmico, Elsevier, Scopus, Springer, Sociedade de gerenciamento de plantas aquáticas além de livros, dissertações e teses. Foram utilizadas as palavras-chave: plantas aquáticas, controle de plantas aquáticas, Salvinia molesta, controle químico, controle químico de plantas aquáticas, tecnologia de aplicação, pulverização eletrostática, monitoramento ambiental, agrotóxicos no ambiente aquático entre outros. A pesquisa foi realizada entre os anos de 2019 e 2020.

\section{Características da planta aquática Salvinia molesta}

A planta aquática Salvinia molesta (D.S. Mitchell), conhecida como salvínia gigante, pertence à família Salvineaceae nativa do sudeste do Brasil, foi encontrada fora do seu local de origem em 1939 no Sri Lanka e se tornou um problema devido à infestação em lavouras de arroz, reduzindo o fluxo dos canais de irrigação e bloqueando a navegação nos canais de transporte (Gettys et al., 2014). É uma planta invasora agressiva e entrou na lista das 100 espécies exóticas que causam prejuízos aos ambientes aquáticos em todo o mundo, em países como Austrália, Índia, Indonésia, África do Sul, Estados Unidos, Zâmbia, Malásia, Cingapura, Papua Nova Guiné, Nova Zelândia, Fiji, Cuba, Trinidad e Tobago, Bornéu, Columbia, Guiana,
Filipinas e Porto Rico entre outros (Gettys et al., 2014; Miranda \& Schwartsburd, 2019).

Incialmente vendida como planta ornamental, S. molesta se propagou rapidamente devido ao fácil cultivo, elevada taxa de crescimento e reprodução vegetativa (Mitchell 1978; Room \& Thomas 1986). Essas características resultam em rápida cobertura da superfície dos corpos hídricos, prejudicando a flora e a fauna locais, além dos usos múltiplos da água (Miranda \& Schwartsburd, 2019).

O crescimento excessivo das plantas aquáticas em geral, especialmente de $S$. molesta, pode causar impactos ecológicos, econômicos e de saúde pública (Brundu, 2015; Sartain \& Mudge, 2018) e, com isso, alterar a composição da comunidade de plantas aquáticas, reduzir a riqueza e abundância das espécies de micro e macroinvertebrados, reduzir o oxigênio da água, impedir os fluxo dos rios, dificultar o transporte e navegação, prejudicar as atividades de esportes aquáticos, reduzir a geração de energia elétrica e a disponibilidade da água nos sistemas de irrigação e drenagem (Hussner, 2014; Gettys et al., 2014; Hussner et al., 2017), além de interferir nos ciclos bioquímicos de nitrogênio e fósforo com alteração na sedimentação, retenção de nutrientes e nas características físicas e químicas da água (Santos et al., 2020).

S. molesta é caracterizada como uma samambaia aquática flutuante que possui tricomas (pelos) na superfície foliar juntamente com outras três espécies de salvínias: S. auriculata, S. herzogii e S. bilboa (McFarland et al., 2004) (Figura 1a). Estas plantas formam colônias flutuantes unidas por um caule horizontal embaixo da superfície da água. As colônias contêm três folhas, sendo duas flutuantes, que possuem tricomas capazes

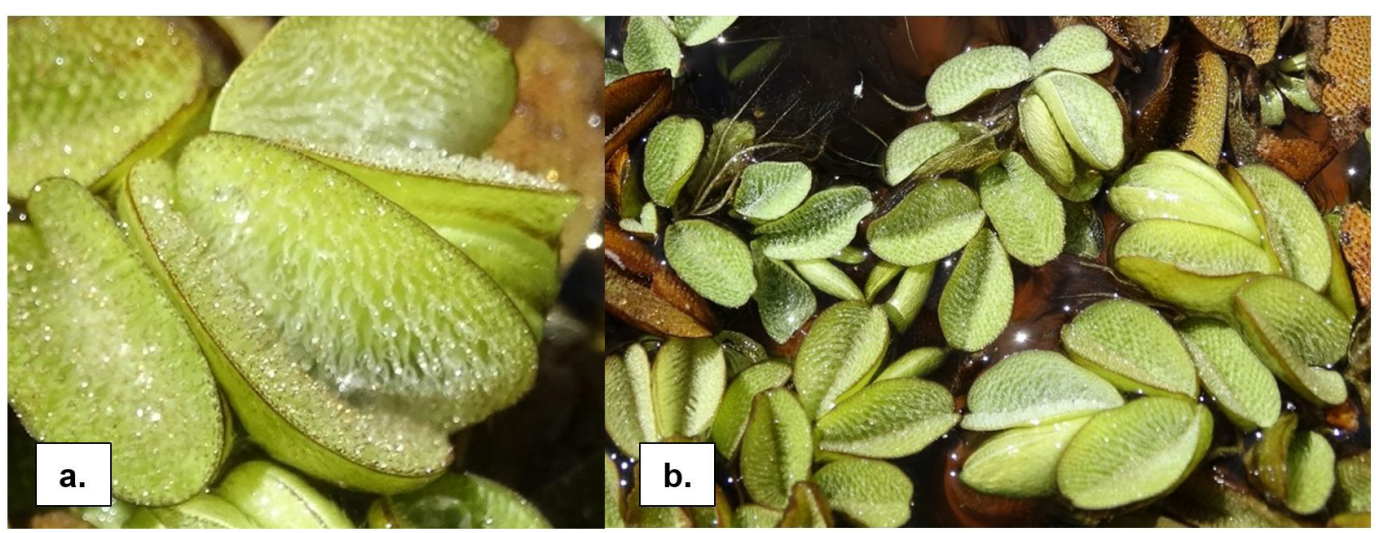

Figura 1. Tricomas (pelos) na superfície foliar de S. molesta (a). Plantas de S. molesta (b). 
de repelir água e auxiliar na flutuação, e uma terceira folha modificada submersa dissecada que absorve os nutrientes na coluna d'água; e possuem esporos entre os filamentos das folhas submersas que se desenvolvem na fase de crescimento ou condições precárias de nutrientes (Galam et al., 2015) (Figura 1b).

A reprodução ocorre por fragmentação: a planta-mãe se quebra e forma mais de uma plantafilha, sendo o meio principal de disseminação da espécie que pode ser influenciada pela atividade dos animais, humanos ou ambiente com a corrente de ventos e ondas (Sartain, 2018).

A planta possui três formas de crescimento: primário, secundário e terciário. $\mathrm{O}$ crescimento primário ocorre com plantas isoladas ou espaçadas com folhas pequenas e ovais de $10 \mathrm{a} 15 \mathrm{~mm}$ de largura e ficam planas sobre a superfície da água (Harley \& Mitchel, 1981). No estágio secundário, o crescimento do caule e entrenós são mais longos e mais vigorosos, as folhas medem de 20 a 50 $\mathrm{mm}$, começam a cobrir a superfície da água e a superfície da terceira folha modificada entra em contato com a água (McFarland et al., 2004). No estágio terciário, ocorre a formação dos tapetes de massa vegetativa na superfície da água com folhas grandes de até $60 \mathrm{~mm}$, que, ao crescerem, são direcionadas para cima formando aglomerados e duas ou mais camadas de plantas que podem chegar até um metro de espessura na coluna d'água (McFarland et al., 2004; Sartain, 2018).

A Salvinia molesta tem crescimento muito rápido e sua biomassa pode dobrar em condição de estufa em 2,2 dias (Cary \& Weerts, 1983). Em campo, sua biomassa vegetal pode dobrar num período de 1 a 8 dias (Finlayson, 1984; Room, 1986; McFarland et al., 2004) (Figura 2a and 2b).

Diante dos efeitos negativos do crescimento excessivo desta macrófita, uma análise de risco foi realizada em alguns países da Europa, na Nova Zelândia e nos Estados Unidos e S. molesta está na lista de espécies exóticas que precisam ser controladas (Hussner et al., 2017).

\section{Controle químico de plantas aquáticas}

O controle químico consiste em usar produtos que causem intoxicação e a morte da planta considerada praga (Pompeo, 2008). Os herbicidas fornecem um conjunto de opções de controle e podem ser aplicados para redução ou remoção de uma espécie alvo ou grupo de plantas alvo em corpos hídricos, incluindo canais de irrigação, rios e lagoas em aplicações parciais ou totais (Getsinger et al., 2014; Netherland, 2014). A seletividade para a planta alvo depende do modo de ação do produto, concentração, tempo de exposição e método de aplicação (Haller, 2014; Hussner et al., 2017).

Práticas de manejo com a rotação de dois ou mais herbicidas, em aplicação sequencial, têm sido estudadas, para melhorar a eficácia no controle de plantas de difícil controle como a S. molesta, para reduzir as chances de estabelecer populações de plantas resistentes aos herbicidas no ambiente aquático (Sartain \& Mudge 2018). A aplicação sequencial é necessária para que ocorra melhor eficácia no controle de $S$. molesta, conforme relatado por Nelson et al. (2007); Cruz et al. (2017); Mudge et al. (2016), além da mistura de herbicidas (Glomski \& Mudge, 2013).

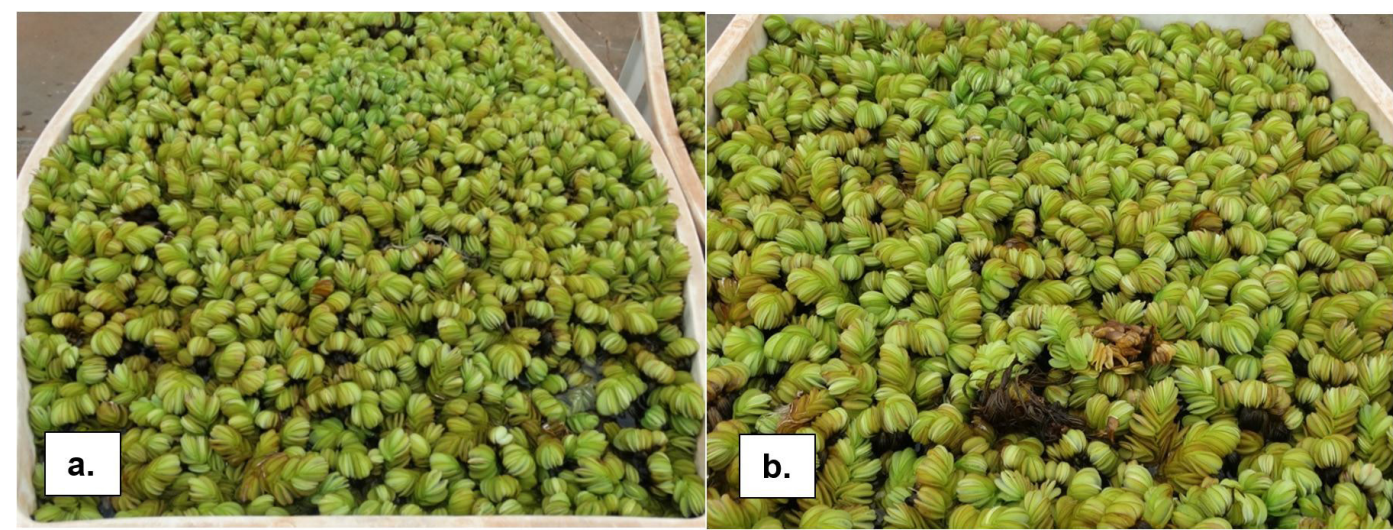

Figura 2. Aglomerados de plantas de S. molesta na superfície da água em condição de microcosmo (a e b). 
Os herbicidas registrados para o controle de plantas aquáticas, em diversos países são: 2,4-D, bispiribac-sódio, carfentrazona-etil, produtos à base de cobre, diclobenil, diquat, endothall, flumioxazina, fluridona, glifosato, haloxifope-metil, imazapyr, imazamox, metslfuron-methyl, penoxsulam, terbutrina e triclopir (Hussner et al., 2017). Em geral, os herbicidas atuam na redução da biomassa das plantas nos ecossistemas aquáticos e, quando utilizados de acordo com as especificações técnicas do produto, podem ser mais eficientes, eficazes e mais econômicos do que o controle mecânico de plantas aquáticas (Netherland, 2014).

Para o controle de $S$. molesta, a utilização de alguns herbicidas tem apresentado resultados de moderados a bons (Mudge et al., 2016), e vários estudos de eficácia já foram realizados com imazapyr (Martins et al., 1999; Nelson et al., 2001; Martins et al., 2002; Emerine et al., 2010; Cruz et al., 2015b), imazamox (Emerine et al., 2010; Campos, 2012; Mudge \& Netherland, 2014), penoxsulam (Wersal \& Madsen, 2010; Mudge et al., 2012; Mudge, 2013; Mudge \& Netherland, 2014), carfentrazone-ethyl (Carvalho et al., 2005; Glomski \& Getsinger, 2006; Mudge et al., 2016), flumioxazina (Mudge, 2013; Mudge et al. 2016; Sartain \& Mudge, 2019); bispyribac-sodium (Glomski \& Mudge, 2014), 2,4-D (Martins et al., 1999), Diquat (Martins et al., 2002; Mudge et al., 2016; Sartain \& Mudge, 2019), glyphosate (Fairchild et al., 2002; Nelson et al., 2007; Cruz et al., 2015a; Mudge et al., 2016; Cruz et al., 2017; Sartain \& Mudge, 2019), endothall (Mudge et al., 2016), fluridone (Mudge et al., 2012), bensufuron, halossulfuron, metsulfuron, rimsulfuron, sulfometuron, tripoxysulfuron (Sartain \& Mudge, 2018).

Dentre as classes de herbicidas que podem ser utilizadas para o controle de $S$. molesta, estão os inibidores da enzima acetolactato sintase (ALS), também conhecida como acetohidroxi ácido sintase (AHAS), enzima fundamental na síntese dos aminoácidos valina, leucina e isoleucinas que são amplamente utilizados na agricultura brasileira (Jursík et al., 2010) e possuem registros em outros países. Estes herbicidas possuem elevada eficácia, amplo espectro de controle de espécies e são utilizados em baixas dosagens para o controle de plantas daninhas (Tranel \& Wright, 2002).

O herbicida imazamox é do grupo químico das imidazolinonas, pertencente aos inibidores ALS e contém um grupo funcional metoximetil no anel piridina (Tan et al., 2005). É absorvido pela folha e translocado para o xilema e floema, causando injúrias e a morte do meristema apical das plantas (Senseman, 2007). Este é eficaz para uma variedade de monocotiledôneas e eudicotiledôneas (Nelson \& Renner, 1998; Tan et al., 2005). No ambiente, não é tóxico para invertebrados aquáticos, abelhas, peixes, aves, mamíferos e pouco tóxico para as algas, não bioacumula, sua meia-vida é de 5 a 15 dias, dependendo da luminosidade e profundidade do corpo hídrico, e a sua dissipação ocorre por diluição e fotólise (Sera, 2010).

No Brasil, a aprovação da resolução CONAMA $n^{\circ} 467$ de 16 de julho de 2015 para uso de produtos químicos em corpos hídricos foi publicada no diário oficial da união $\mathrm{n}^{\circ} 135$ de 17 de julho de 2015, seção 1, pág. 70 a 71 que "dispõe sobre critérios para autorização de uso de produtos ou de agentes de processos físicos, químicos ou biológicos para o controle de organismos ou contaminantes em corpos hídricos superficiais e da outras providências" (Brasil, 2015). E o herbicida imazamox está em processo de avaliação para ser registrado e autorizado para utilização nos corpos brasileiros (Brasil, 2020).

\section{Tecnologias para aplicação de herbicidas}

Os herbicidas são depositados na superfície dos vegetais, podendo ser absorvidos em maior ou menor quantidade para exercerem seu efeito internamente (Kissmann, 1998; Queiroz et al., 2008). Porém, a superfície dos vegetais apresenta uma barreira física, denominada de cutícula, variável entre as espécies, o que pode dificultar a penetração de soluções aquosas (Queiroz et al., 2008).

Para vencer esta barreira, podem ser utilizados alguns itens na aplicação, tais como: adjuvantes, tecnologias e a aplicação nas condições favoráveis que fazem com que o produto alcance o alvo na concentração correta, com o mínimo de perda e com menor risco de contaminação do ambiente (Matuo, 1990; Silva et al., 2014).

Assim, visando manter a efetividade do herbicida, têm sido adicionados adjuvantes às caldas fitossanitárias, o que pode alterar as características físico-químicas, dependendo do tipo e da composição das formulações (Iost \& Raetano, 2010). Estas alterações, se bem compreendidas e selecionadas, interferem positivamente no diâmetro e uniformidade das gotas, colaborando para a colocação do produto no alvo e, consequentemente, melhorando a eficiência do ingrediente ativo e possível redução de perdas por deriva (Matuo, 1990). 
Os adjuvantes atuam sobre o ingrediente ativo por vários mecanismos como: redução da tensão superficial da gota, facilidade da emulsificação, dispersão, aderência e ação umectante do herbicida, alteração da disponibilidade na gota para sua mobilidade e absorção através do tecido vegetal (Devendra et al., 2004).

A adição de adjuvantes aos herbicidas vem sendo estudada no controle de $S$. molesta para melhorar o desempenho no controle desta macrófita, tais como: endothall- $\mathrm{K}+0,5 \%$ cide-kick, endothall amine $+0,5 \%$ cide-kick, diquat $+0,5 \%$ cide-kick e glyphosate $+0,5 \%$ cide-kick (Nelson et al., 2001); glyphosate $+0,25 \%$ de LI- $700^{\circledR}$, glyphosate $+0,25 \%$ Cygnet $^{\circledR}$, glyphosate $+0,25 \%$ Kinetic $^{\circledR}$, glyphosate $+0,25 \%$ Optima $^{\circledR}$ e glyphosate $+0,25 \%$ Mon $0818^{\circledR}$ (Fairchild et al., 2002); penoxsulam $+0,5 \%$ Sunwet $^{\circledR}$ (Wersal \& Madsen, 2010); glyphosate + $0,5 \%$ de Aterbane ${ }^{\circledR}$ (Cruz et al., 2015a); glyphosate + óleo vegetal (Mudge et al., 2016); saflufenacil, flumiclorac e glufosinato $+0,25 \% \mathrm{vv}^{-1}$ de mistura de óleo vegetal modificado e surfactante não iônico organossiliconado (Sartain \& Mudge, 2018); diquat + $0,1 \% \mathrm{vV}^{-1}$ de surfactante não iônico organossiliconado (Sartain \& Mudge, 2019).

Para melhorar a eficiência da aplicação de herbicidas isolados ou acrescidos de adjuvantes para $S$. molesta, é necessário utilizar tecnologias de aplicação que aumentem a deposição do produto diretamente no alvo e que não causem a contaminação do ambiente aquático (Serra et al., 2008).

$\mathrm{Na}$ aplicação convencional, as forças que atuam nas gotas são a gravidade e a inércia (Martin \& Latheef, 2017) e mais de $90 \%$ dos pesticidas são aplicados com sistemas de bicos de pulverização hidráulicos (Figura 3a and 3b), que são mais propensos à deriva, devido à não uniformidade do tamanho das gotas, e a eficiência na deposição no alvo é inferior a 30\% (Sande et al., 2008). As gotas menores são perdidas pela evaporação ou deriva e as maiores, devido à sedimentação gravitacional, pelo solo (Patel, 2016).

O emprego de gotas finas proporciona melhor cobertura de alvos no controle de problemas fitossanitários, porém gotículas com pequenas massas possuem pouca energia cinética e causam efeito de deriva no processo de captura pelo alvo. Para que essas pequenas gotas sejam coletadas pelo alvo com menores impactos ambientais, podem ser acrescentadas forças externas como o aumento da velocidade de lançamento com maiores pressões de trabalho ou o aumento da velocidade terminal com cargas elétricas (Chaim, 2006).

A pulverização eletrostática trata-se de uma técnica na qual ocorrem transferências de cargas elétricas para as gotas, a fim de promover a atração com a planta, devido à diferença de potencial elétrico (Figura 4a and 4b). Esta tecnologia pode melhorar o depósito das gotas finas na planta com menor contaminação ambiental, o que permite a redução do volume de calda e dos custos da pulverização (Zheng et al., 2002).

$\mathrm{Na}$ aplicação em sistema eletrostático com gotas com tamanho de 100 micrometros e eletrificadas (Chaim, 2006), a calda de aplicação é carregada eletricamente formando gotas carregadas (Pan et al., 2019) e a planta alvo possui carga neutra e, quando as gotas se aproximam dela, sua carga negativa fica em direção oposta e as folhas ficam com carga positiva, o que atrai as gotas para toda a superfície

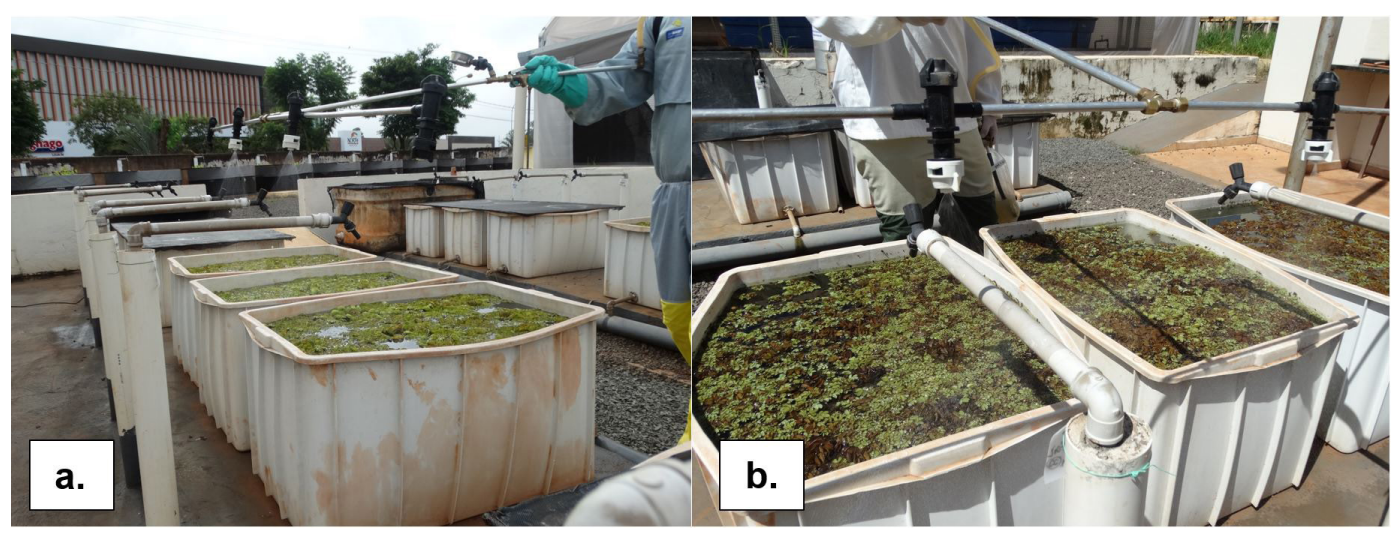

Figura 3. Aplicação de herbicida com bicos de pulverização hidráulicos em condição de microcosmos (a e b), do Laboratório de Ecotoxicologia e Eficácia dos Agrotóxicos, LEEA, ao Centro Universitário da Fundação Educacional de Barretos, estrutura adquirida pelo projeto de auxílio pesquisa da Fundação de Ampara à Pesquisa do Estado de São Paulo, Fapesp, proc. N. 2015/16735-5. 
foliar tanto na parte abaxial como adaxial (He et al., 2011; Salcedo et al., 2020). Esta tecnologia resulta em maior cobertura dos alvos, com menor perda por deriva e, consequentemente, menor desperdício de produto e menor contaminação ambiental dos corpos hídricos.

A tecnologia utilizada no momento da aplicação em plantas daninhas aquáticas é muito importante, pois as gotas aplicadas com pulverizadores convencionais podem destinar-se à água diretamente e causar impactos maiores que o tolerado. Enquanto a aplicação eletrostática com atração das gotas pelas plantas com adição ou não de adjuvantes pode tornar a aplicação mais segura, fazendo com que a gota chegue somente na planta alvo de controle (Garlich, 2020).

\section{Monitoramento ambiental de agrotóxicos no ambiente aquático}

O monitoramento ambiental é a melhor forma para compreender os efeitos da aplicação de produtos nos ambientes aquáticos. Os herbicidas aplicados nos corpos hídricos podem sofrer fotodegradação, degradação microbiana, adsorção por matéria orgânica particulada ou argilas em suspensão, diluição e meia-vida de algumas horas e/ou dias (Souza et al., 2017) e o monitoramento do comportamento destes produtos é importante para o conhecimento de sua ação direta ou indireta na qualidade de água e nos organismos não alvos da aplicação.

A simulação de efeitos ambientais em condição de microcosmos ou de mesocosmos (Organisation for Economic Co-operation and Development, 2004) é uma ferramenta útil para avaliar potenciais riscos do efeito da exposição dos organismos não alvos após aplicação de herbicidas e estimar os efeitos nas características físico-químicas da água (Guimarães et al., 2003). Esse tipo de avaliação pode exibir resultados satisfatórios em condições mais próximas do real e estas informações podem auxiliar na tomada de decisão na utilização de herbicidas no ambiente aquático (Figura 5a and 5b).

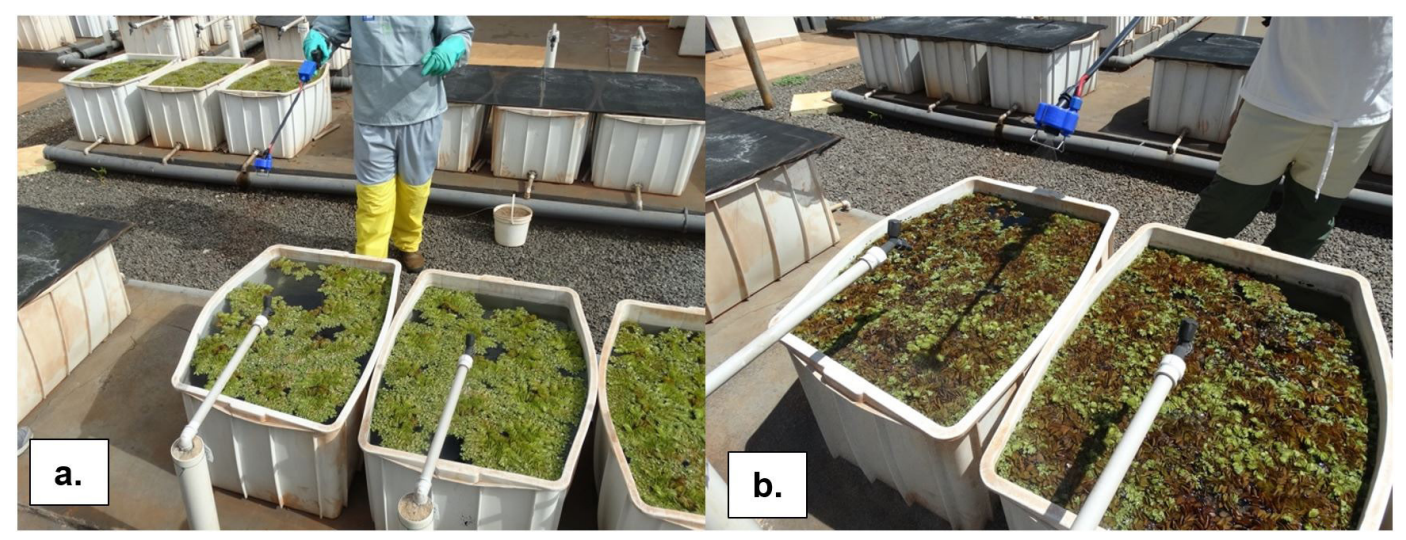

Figura 4. Aplicação de herbicida com pulverizador eletrostático em condição de microcosmos (a e b).

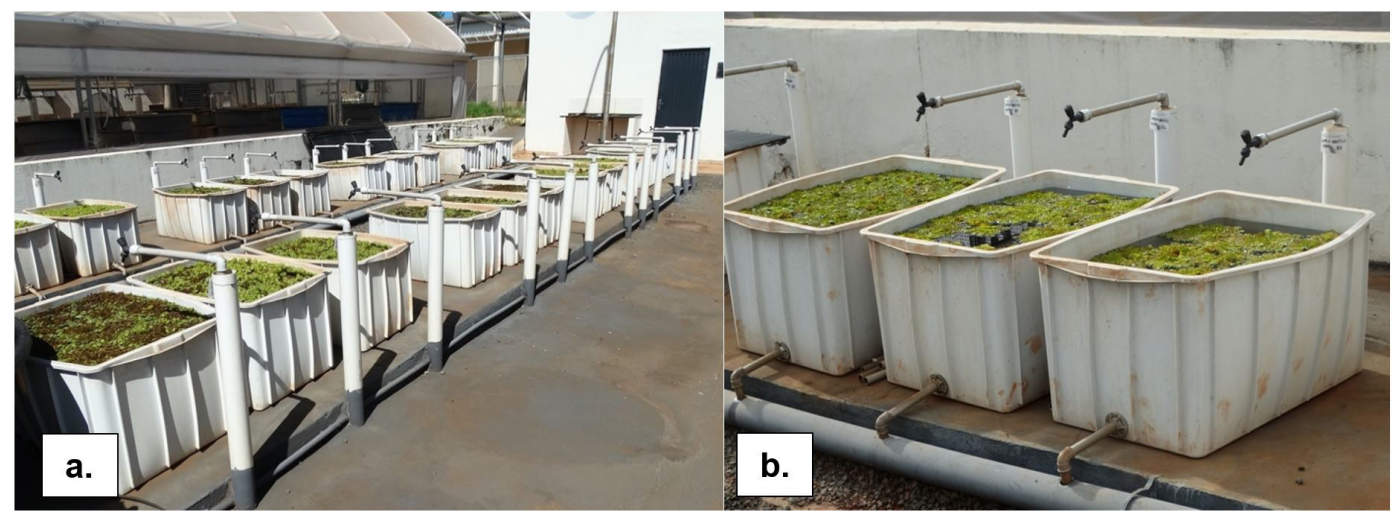

Figura 5. Simulação ambiental em condição de microcosmos (a). Unidade experimental com três caixas plásticas de $200 \mathrm{~L}$ (b). 
Essa análise é importante para preencher a lacuna entre os estudos laboratoriais e de campo e, assim, tentar entender e prever o que pode ocorrer em um ambiente natural sujeito a risco ou na determinação da segurança de produtos químicos. Todo estudo em condição de microcosmos ou mesocosmos deve ser planejado e testar uma hipótese usando informações obtidas na literatura e em etapas anteriores de avaliação de risco (Campbell et al., 1999; Giddings et al., 2002).

Alguns estudos foram realizados utilizando mesocosmos para determinar os efeitos em indicadores ambientais de qualidade de água, tais como: estudo com método de fluxo contínuo de água simulando situação mais próxima do real para demonstrar os possíveis riscos do uso de substâncias químicas no controle de plantas aquáticas (Guimarães et al., 2003), avaliação da eficácia do herbicida diquat no controle da macrófita aquática Eichhornia crassipes e os possíveis efeitos do controle sobre as características de qualidade de água (Martins \& Pitelli, 2005) e a eficácia do herbicida fluridone para controle de plantas aquáticas submersas Egeria najas e E. densa em condição de microcosmo e represa de pequeno porte sem fluxo de água e os efeitos nas características da qualidade de água (Tanaka et al., 2002).

Este modelo de estudo também foi utilizado para avaliar a resposta do polliqueto marinho Sabellaria alveolata exposta ao cloro aquoso usado como agente anti-incrustante na água de resfriamento da usina (Last et al., 2016), para determinar o efeito do inseticida neonicotinoide tiametoxam, que pode atingir áreas úmidas em agroecossistemas e os possíveis efeitos sobre comunidade fito e zooplanctônica (Lobson et al., 2018), para determinar a capacidade de remoção do Cromo hexavalente IV de águas residuárias de mina de extração de níquel pelas plantas Leporonia articulata e Machaerina rubiginosa (Amina et al., 2018) e para avaliação de efeito do herbicida atrazine na reprodução de sapo de críquete (Acris blanchardi) (Hoskins et al., 2019).

\section{Conclusão}

Esta revisão de literatura mostra que estudos de tecnologias para aplicação de herbicidas são importantes, principalmente para manter a sanidade e a estabilidade dos corpos hídricos, devido aos prejuízos causados pela infestação de $S$. molesta. A aplicação de herbicidas com a tecnologia adequada pode ser mais eficaz no controle desta planta e ajudar no processo de resiliência dos corpos hídricos com a diminuição da massa vegetal que é formada. Os estudos de monitoramento ambiental auxiliam na compreensão dos efeitos desta aplicação e são importantes devido à recente aprovação da legislação que autoriza a aplicação de produtos nos corpos hídricos brasileiros. E com isso, colaborar no estabelecimento de um processo padronizado de intervenção química e monitoramento ambiental para que ocorra eficiência no controle das plantas alvo e segurança aos organismos aquáticos.

\section{Agradecimentos}

À Fundação de Amparo à Pesquisa do Estado de São Paulo, pelo auxílio pesquisa processo n. 2015/16735-5, para a instalação da estrutura de microcosmos, sob responsabilidade do Prof. Dr. Claudinei da Cruz, do Laboratório de Ecotoxicologia e Eficácia dos Agrotóxicos, LEEA, pertencente ao Centro Universitário da Fundação Educacional de Barretos.

\section{Referências}

Amina, R., Edraki, M., Doley, D., \& Sheridan, C. (2018). Removal of dissolved chromium from synthetic mine effluent: A mesocosm experiment. The Science of the Total Environment, 637-638, 1252-1261.

Brasil. Conselho Nacional do Meio Ambiente CONAMA. (2015, 17 de julho). Resolução no 465 de 16 de julho de 2015, Dispõe sobre critérios para autorização de uso de produtos ou de agentes de processos físicos, químicos ou biológicos para o controle de organismos ou contaminantes em corpos hídricos superficiais e da outras providências, Diário Oficial [da] República Federativa do Brasil, Brasília.

Brasil. Instituto Brasileiro do Meio Ambiente e dos Recursos Naturais Renováveis. (2020). Registro de Agrotóxicos de Uso Não Agrícola. Recuperado em 23 de outubro de 2020, de http://www.ibama. gov.br/agrotoxicos/quimicos-biologicos-registros/ registro-de-agrotoxicos-de-uso-nao-agricola

Brundu, G. (2015). Plant invaders in European and Mediterranean inland waters: profiles, distribution, and threats. Hydrobiologia, 746, 61-79. 
Campbell, P. J., Arnold, D. J. S., Brock, T. C. M., Grandy, N. C., Heger, W., Heimbach, F., Maund, S. J., \& Streloke, M. (1999). Guidance Document on Higher-tier Aquatic Risk Assessment for Pexticides (HARAP). Brussels, BE: SETAC-Europe.

Campos, C. F. (2012). Influência da chuva após aplicação de imazamox sobre o controle de plantas daninhas aquáticas. Bioscience Journal, 28, 413-419.

Carvalho, F. T. D., Velini, E. D., Negrisoli, E., \& Rossi, C. V. S. (2005). Eficácia do carfentrazoneethyl no controle de plantas aquáticas latifoliadas em caixas-d'água. Planta Daninha, 23, 305-310.

Cary, P. R., \& Weerts, P. G. J. (1983). Growth of Salvinia molesta as affected by water temperature and nutrition. I. Effects of nitrogen level and nitrogen compounds. Aquatic Botany, 16, 163-172.

Chaim, A. (2006). Pulverização eletrostática: principais processos utilizados para eletrificação de gotas (Documento 57, 17 p.). Jaguariúna: Embrapa Meio Ambiente.

Cruz, C., Cervoni, J. H. C., Silva, A. F., Garlich, N., \& Pitelli, R. A. (2017). Aplicação sequencial de Glyphosate em plantas aquáticas de difícil controle em condição de microcosmos. Ciência e Cultura, 13, 59-63.

Cruz, C., Silva, A. F., Luna, L. V., Yamauchi, A. K. F., Garlich, N., \& Pitelli, R. A. (2015a). Glyphosate effectiveness in the control of Macrophytes under a greenhouse condition. Planta Daninha, 33, 24-47.

Cruz, C., Silva, A. F., Shiogiri, N. S., Garlich, N., \& Pitelli, R. A. (2015b). Imazapyr herbicide efficacy on floating macrophytes control and ecotoxicology for non-target organisms. Planta Daninha, 33, 103-108.

Devendra, R., Umamahesh, V., Praad, T. V. R., Prasad, T. G., Asha, S. T., \& Ashok, A. (2004). Influence of surfactants on efficacy of different herbicides in control of Cyperus rotundus and Oxalis latifolia. Current Science, 86, 1148-1151.

Emerine, S. E., Richardson, R. J., True, S. L., West, A. M., \& Roten, R. L. (2010). Greenhouse response of six aquatic invasive weeds to Imazamox. Journal of Aquatic Plant Management, 48, 105-111.
Esteves, F. A. (1998). Fundamentos de limnologia (602 p.). Rio de Janeiro: Interciência/FINEP.

Fairchild, J. F., Allert, A. L., Riddle, J. S., \& Gladwin, D. R. (2002). Efficacy of glyphosate and five surfactants for controlling giant salvinia. Journal of Aquatic Plant Management, 40, 53-58.

Finlayson, C. M. (1984). Growth rates of Salvinia molesta in Lake Moondarra, Mount Isa, Australia. Aquatic Botany, 18, 257-262.

Galam, D., Silva, J., Sanders, D., \& Oard, J. H. (2015). Morphological and genetic survey of Giant Salvinia populations in Louisiana and Texas. Aquatic Botany, 127, 20-25.

Garlich, N. (2020). Controle de salvinia molesta em condição de microcosmo por aplicação eletrostática de herbicida e adjuvante e o efeito sobre bioindicadores aquáticos. Jaboticabal: Faculdade de Ciências Agrárias e Veterinárias, Universidade Estadual Paulista.

Garlich, N., Cruz, C., Silva, A. F., Malaspina, I. C., Ferreira, R. G., Tedesque, M. G., Pitelli, R. A., \& Bianco, S. (2016a). Effectiveness of diquat, copper hydroxide, copper oxychloride and their association in control of submerged macrophytes Ceratophyllum demersum. Planta Daninha, 34(1), 117-123.

Garlich, N., Da Cruz, C., Da Silva, A. F., Carraschi, S. P., Malaspina, I. C., Pitelli, R. A., \& Bianco, S. (2016b). Diquat associated with copper sources for algae control: efficacy and ecotoxicology. Journal of Environmental Science and Health. Part. B, Pesticides, Food Contaminants, and Agricultural Wastes, 51(4), 215-221.

Getsinger, K. D., Dibble, E., Rodgers, J. H., \& Spencer, D. (2014). Benefits of controlling nuisance aquatic plants and Algae in the United States (12 p.). AMES: CAST.

Gettys, L. A., Haller, W. T., \& Petty, D. G. (2014). Biology and control of aquatic plants. A best management practices handbook (3rd ed., 238 p.). Mariett, GA: Aquatic ecosystem restoration foundation.

Giddings, J. M., Brock, T. C. M., Heger, W., Heimbach, F., Maund, S. J., Norman, S. M., Ratte, H. T., Schäfers, C., \& Streloke, M. (2002). Community- 
level aquatic system studies-interpretation criteria. In Proceedings from the CLASSIC Workshop held at the Fraunhofer Institute (44 p.). 30 May-2June, Smallenberg, Germany: SETAC PRESS.

Glomski, L. A. M., \& Getsinger, K. D. (2006). Carfentrazone-ethyl for Control of Giant Salvinia. Journal of Aquatic Plant Management, 44, 136-138.

Glomski, L. M., \& Mudge, C. R. (2013). Effect of subsurface and foliar applications of bispyribacsodium on water hyacinth, water lettuce, and giant salvinia. Journal of Aquatic Plant Management, 51, 62-65.

Glomski, L. M., \& Mudge, C. R. (2014). Effect of subsurface and foliar applications of bispyribacsodium on water hyacinth, water lettuce, and giant salvinia. Journal of Aquatic Plant Management, 51, 62-65.

Guimarães, G. L., Foloni, L. L., Pitelli, R. A., \& Martins, A. T. (2003). Metodologia para avaliação de impacto ambiental de macrófitas em mesocosmos. Planta Daninha, 21, 37-42.

Haller, W. (2014). Aquatic herbicide application methods. In L. Gettys, W. Haller, \& D. Petty (Eds.), Biological Control of Aquatic Plants. A best management practices handbook (3rd ed., pp. 193-198). USA: Aquatic Ecosystem Restoration Foundation.

Harley, K. L. S., \& Mitchel, D. S. (1981). The biology of Australian weeds, 6. Salvinia molesta D.S. Mitchell. Journal of the Australian Institute of Agricultural Science, 47, 67-76.

He, X., Zeng, A., Liu, Y., \& Song, J. (2011). Precision orchard sprayer based on automatically infrared target detecting and electrostatic spraying techniques. International Journal of Agricultural and Biological Engineering, 4, 35-40.

Hoskins, T. D., Dellapina, M., Mapoulias, D. M., $\&$ Boone, M. D. (2019). Effects of larval atrazine exposure in mesocosms on Blanchard'scricket frogs (Acris blanchardi) reared through overwintering and to reproductive age. Chemosphere, 220, 845-857.

Hussner, A. (2014). Long-term macrophyte mapping documents a continuously shift from native to non-native aquatic plant dominance in the thermally abnormal River Erft (North RhineWestphalia, Germany). Limnologica, 48, 39-45.

Hussner, A., Stiers, I., Verhofstad, M. J. J. M., Bakker, E. S., Grutters, B. M. C., Haury, J., Valkenburg, J. L. C. H. V., Brundu, G., Newman, J., Clayton, J. S., Anderson, L. W. J., \& Hosfstra, D. (2017). Management and control methods of invasive alien freshwater aquatic plants: a review. Aquatic Botany, 136, 112-137.

Iost, C. A., \& Raetano, C. G. (2010). Tensão superficial dinâmica e ângulo de contato de soluções aquosas com surfactantes em superfícies artificiais e naturais. Engenharia Agrícola, 30, 670-680.

Jursík, M., Soukup, J., \& Holec, J. (2010). Herbicide mode of actions and symptoms of plant injury by herbicides: inhibitors of acetolactate synthase (ALS inhibitors). Listy Cukrovarnické a Reparské, 126, 376-379.

Kissmann, K. G. (1998). Adjuvantes para caldas de produtos fitossanitários. In J. V. C. Guedes, \& S. H. B. Dornelles (Ed.), Tecnologia e segurança na aplicação de agrotóxicos: novas tecnologias (pp. 95-104). Santa Maria: Departamento de Defesa.

Last, K. S., Hendrick, V. J., Beveridge, C. M., Roberts, D. A., \& Wilding, T. A. (2016). Lethal and sub-lethal responses of the biogenic reef forming polychaete Sabellaria alveolate to aqueous chlorine and temperature. Marine Environmental Research, 117, 44-53.

Lobson, C., Luong, K., Seburn, D., White, M., Hann, B., Prosser, R. S., Wongb, C. S., \& Hanson, M. L. (2018). Fate of thiamethoxam in mesocosms and response of the zooplankton community. The Science of the Total Environment, 637-638, 1150-1157.

Maria, M. A., Lange, L. C., Castro, S. R., Soares, A. C., \& Meyer, S. T. (2018). Evaluation of glyphosate effect concentration to control Eichhornia crassipes and Salvinia sp. Engenharia Sanitaria e Ambiental, 23(5), 881-889.

Martin, D. E., \& Latheef, M. A. (2017). Efficacy of electrostatically charged glyphosate on ryegrass. Journal of Electrostatics, 90, 45-53.

Martins, A. T., \& Pitelli, R. A. (2005). Efeitos do manejo de Eichhornia crassipes sobre a qualidade 
da água em condições de mesocosmos. Planta Daninha, 23, 233-242.

Martins, D., Velini, E. D., Cavenaghi, A. L., \& Mendonça, C. G. D. (1999). Controle químico de plantas daninhas aquáticas em condições controladas - Caixa d'água. Planta Daninha, 17, 289-296.

Martins, D., Velini, E. D., Negrisoli, E., \& Tofoli, G. R. (2002). Controle químico de Pistia stratiotes, Eichhornia crassipes E Salvinia molesta em caixas d'água. Planta Daninha, 20, 83-88.

Matuo, T. (1990). Técnicas de aplicação de defensivos agrícolas (139 p.). Jaboticabal: Funep.

McFarland, D. G., Nelson, L. S., Grodowitz, M. J., Smart, R. M., \& Owens, C. S. (2004). Salvinia molesta D.S. Mitchell (Giant Salvinia) in the United States: A review of species ecology and approaches to management. Washington, DC: U.S. Army Corps of Engineer.

Miranda, C. V., \& Schwartsburd, P. B. (2019). Salvinia (Salviniaceae) in southern and southeastern Brazil: including new taxa, new distribution records, and new morphological characters. Brazilian Journal of Botany, 42, 171-188.

Mitchell, D. S. (1978). Aquatic weeds in Papua New Guinea. Science in New Guinea, 6, 154-160.

Mudge, C. R. (2013). Interactions of herbicides, surfactants, and the giant salvinia weevil (Cyrtobagous salviniae) for control of giant salvinia (Salvinia molesta). Journal of Aquatic Plant Management, 51, 77-87.

Mudge, C. R., \& Netherland, M. D. (2014). Response of invasive floating plants and nontarget emergent plants to foliar applications of imazamox and penoxsulam. Journal of Aquatic Plant Management, 52, 1-7.

Mudge, C. R., Heilman, M. A., Theel, H. J., \& Getsinger, K. D. (2012). Efficacy of subsurface and foliar penoxsulam and fluridone applications on giant salvinia. Journal of Aquatic Plant Management, 50, 116-124.

Mudge, C. R., Perret, A. J., \& Winslow, J. R. (2016). Evaluation of foliar herbicide and surfactant combinations for control of giant salvinia at three application timings. Journal of Aquatic Plant Management, 54, 32-36.

Nelson, K. A., \& Renner, K. A. (1998). Weed control in wide- and narrow-row soybean (Glycine max) with imazamox, imazethapyr, and CGA-277476 plus quizalofop. Weed Technology, 12, 137-144.

Nelson, L. S., Glomski, L. M., \& Gladwin, D. N. (2007). Effect of Glyphosate Rate and spray volume on control of Giant Salvinia. Journal of Aquatic Plant Management, 45, 58-61.

Nelson, L. S., Skogerboe, J. G., \& Getsinger, K. D. (2001). Herbicide evaluation against giant salvinia. Journal of Aquatic Plant Management, $39,48-53$

Netherland, M. D. (2014). Chemical control of aquatic weeds. In L. Gettys, W. Haller, \& D. Petty (Eds.), Biological Control of aquatic plants. A best management practices handbook (3rd ed., pp. 71-88). USA: Aquatic Ecosystem Restoration Foundation.

Organisation for Economic Co-operation and Development - OECD. (2004). Draft Guidance document on simulated freshwater lentic field tests (Outdoor Microcosms and Mesocosms). 26 p. Recuperado em 23 de outubro de 2020 , de http://www.oecd.org/fr/securitechimique/ essais/32612239.pdf

Pan, X. L., Dong, F. S., Wu, X. H., Xu, J., Liu, X. G., \& Zheng, Y. Q. (2019). Progress of the discovery, application, and control technologies of chemical pesticides in China. Journal of Integrative Agriculture, 18, 840-853.

Patel, M. K. (2016). Technological improvements in electrostatic spraying and its impact to agriculture during the last decade and future research perspectives-A review. Engineering in Agriculture, Environment and Food, 9(1), 92-100.

Pitelli, R. A., Pitelli, R. L. C. M., Filho, W. R. C., Rocha, R. J. S., Cruz, F., \& Neto, J. J. (2018). Avaliação das concentrações de nutrientes e metais pesados nas principais macrófitas aquáticas do reservatório de Santana-RJ no período de três anos. Semioses: Inovação, Desenvolvimento e Sustentabilidade, 12(2), 112-126. 
Pompeo, M. (2008). Monitoramento e manejo de plantas aquáticas. Oecologia Brasiliensis, 12, 406-424.

Queiroz, A. A., Martins, J. A. S., \& Cunha, J. P. A. R. (2008). Adjuvantes e qualidade da água na aplicação de agrotóxicos. Bioscience Journal, 24, 8-19.

Room, P. M. (1986). Salvinia molesta-a floating weed and its biological control In R. L. Kitching (Ed.), The ecology of exotic animals and plants (pp. 164-186). Brisbane, Queensland: John Wiley.

Room, P. M., \& Thomas, P. A. (1986). Nitrogen, phosphorus and potassium in Salvinia molesta Mitchell in the field: effects of weather, insect damage, fertilizers and age. Aquatic Botany, 24, 213-232.

Salcedo, R., Llop, J., Campos, J., Costas, M., Gallart, M., Ortega, P., \& Gil, E. (2020). Evaluation of leaf deposit quality between electrostatic and conventional multi-row sprayers in a trellised vineyard. Crop Protection (Guildford, Surrey), 127, 104964.

Sande, J. C. V., Huijsmans, J. F. M., Porskamp, H. A. J., Michielsen, J. M. G. P., Stalinga, H., Holterman, H. J., \& Jong, A. (2008). Spray tecniques: how to optimise spray deposition and minimize spray drift. The Environmentalist, 28, 9-17.

Santos, N. B. C., Arruda, E. C. P., Neto, A. G. B., \& Oliveira, A. F. M. (2020). Assessing the effects of water quality on leaf morphoanatomy, ultrastructure and photosynthetic pigment content of Salvinia auriculata Aubl.(Salviniaceae). Ecotoxicology and Environmental Safety, 190, 110061.

Sartain, B. T. (2018). Exploring alternative giant salvinia (Salvinia molesta D.S. Mitchell) management strategies (Doctoral Dissertations). Louisiana State University and Agricultural and Mechanical College. 121 p. Recuperado em 23 de outubro de 2020, de https://digitalcommons. lsu.edu/gradschool_dissertations/4542

Sartain, B. T., \& Mudge, C. R. (2018). Evaluation of 12 foliar applied non-aquatic herbicides for efficacy against giant salvinia (Salvinia molesta). Journal of Aquatic Plant Management, 56, 107-112.
Sartain, B. T., \& Mudge, C. R. (2019). The response of giant salvinia to foliar herbicide applications at three winter timings. Journal of Aquatic Plant Management, 57, 1-6.

Senseman, S. A. (2007). Herbicide handbook (9th ed., 458 p.). Lawrence, KS: Weed Science Society of America.

Serra, M. E., Chaim, A., \& Raetano, C. G. (2008). Pontas de pulverização e eletrificação das gotas na deposição da calda em plantas de crisântemo. Pesquisa Agropecuária Brasileira, 43, 479-485.

Silva, B. M., Ruas, R. A. A., Sichocki, D., Dezordi, L. R., \& Caixeta, L. F. (2014). Deposição da calda de pulverização aplicada com pontas de jato plano em diferentes partes da planta de soja (glycinemax) e milho (zeamays). Revista Engenharia na Agricultura, 22, 17-24.

Silva, D. S., Marques, E. E., \& Lólis, S. F. (2012). Macrófitas aquáticas:"vilãs ou mocinhas"? Revista Interface, 4, 17-27.

Souza, E. L. C., Foloni, L. L., Filho, J. T., Velini, E. D., Siono, L. M., \& Silva, J. R. M. (2017). Half-Life of Glyphosate on the Control of Water Hyacinths in Water Tanks. Journal of Water Resource and Protection, 9, 470-481.

Syracuse Environmental Research Associates - SERA. (2010). Imazamox: Human Health and Ecological Risk Assessment. Final Report. Recuperado em 23 de outubro de 2020, de http:// www.fs.fed.us/foresthealth/pesticide/pdfs/052-2402a_Imazamox.pdf

Tan, S., Evans, R. R., Dahmer, M. L., Singh, B. K., \& Shaner, D. L. (2005). Imidazolinone-tolerant crops: history, current status and future. Pest Management Science, 61, 246-257.

Tanaka, R. H., Velini, E. D., Martins, D., Bronhara, A. A., Silva, M. A. S., Cavenaghi, A. L., \& Tomazela, M. S. (2002). Avaliação de herbicidas para o controle de egéria em laboratório, caixa d'água e represa sem fluxo de água. Planta Daninha, 20, 73-81.

Tranel, P., \& Wright, T. R. (2002). Resistance of weeds to ALS-inhibiting herbicides: what have we learned? Weed Science, 50, 700-712. 
Wersal, R. M., \& Madsen, J. D. (2010). Combinations of Penoxsulam and Diquat as foliar applications for control of Waterhyacinth and Common Salvinia: Evidence of herbicide antagonism. Journal of Aquatic Plant Management, 48, 21-25.
Zheng, J., Zhou, H., \& Xu, Y. (2002). Advances in pesticide electrostatic spraying in China. Transactions of the ASAE. American Society of Agricultural Engineers, 34, 1-12. 